\title{
Neonatal sepsis for postpartum depression
}

\author{
Chintha Jayasinghe ${ }^{1}$ \& Chrishantha Abeysena ${ }^{2 *}$ \\ ${ }^{1}$ Epidemiology Unit, Ministry of Health, Sri Lanka; ${ }^{2}$ Department of Public Health, Faculty of Medicine, \\ University of Kelaniya, Sri Lanka \\ "Correspondence: chinthaj@yahoo.com (iD https://orcid.org/0000-0002-0476-1785 \\ DOI: https://doi.org/110.4038/jccpsl.v25i3.8194
}

Received on: 29 January 2019

Accepted on: 29 July 2019

\begin{abstract}
Introduction: The postpartum period is generally a time of happiness and bonding between mother and the newborn, but complicated pregnancies can alter this. Postpartum depression (PPD) is a common complication of childbirth.

Objectives: To determine the effect of neonatal sepsis on PPD of mothers

Methods: This was a descriptive study conducted in secondary and tertiary care hospitals in the district of Gampaha. The study population comprised postpartum mothers of neonates who were diagnosed with sepsis $(n=236)$ and those who were not $(n=240)$. Neonatal sepsis was defined by the presence of one of the clinical signs according to the IMCI strategy or culture positive blood or cerebrospinal fluid. The validated Edinburgh Postpartum Depression Scale and an interviewer-administrated questionnaire were used. Multiple logistic regression was applied and adjusted odds ratios (aOR) and 95\% confidence intervals (CI) were calculated.

Results: The study revealed that among the mothers of sepsis neonates, 185 (78.4\%) were depressed. There were $51(21.2 \%)$ mothers also depressed among the mother of non-sepsis babies. The association between PPD and neonatal sepsis was statistically significant $(\mathrm{aOR}=13.44 ; 95 \% \mathrm{CI}=8.68,20.83)$. The socio-demographic factors, obstetric and neonatal factors were not independently associated with PPD.

Conclusions: Postpartum depression among mothers of sepic neonates was higher than in mothers of apparently healthy neonates. It is essential to arrange a screening programme and counselling sessions while in the hospital for all the mothers.
\end{abstract}

Key words: counselling, neonates, prevention, risk factors, sepsis 


\section{Introduction}

Unpleasant psychological nature of the parents, especially of mothers having sick neonates is well documented in literature (1-2). Some authors have measured it as stressors (3-4), whereas others have measured it as depression (5-6). Identifying these psychological conditions is important to improve the ability of mothers to meet the needs of their babies and to develop the skills required to fulfil their role. Mothers in the postpartum period are a special group whose psychological changes are measured as postpartum depression (PPD).

The non-psychiatric depression that women experience during the first four weeks of the postpartum period is overall referred to as PPD (7). Risk factors for PPD have been examined indicating that the strongest predictors for PPD include depression or anxiety during pregnancy, personal and family history of depression, lack of social support and stressful life events (8-10). However, most women who were depressed did not necessarily have personal or family histories of depression. It is therefore important to consider causes that occur more commonly, such as stressful life events including sickness of the neonates.

The postpartum period is generally a time of happiness and bonding between the mother and the newborn, but complicated pregnancies can alter this. PPD is a common complication of childbirth. Studies carried out to find out the prevalence of depression among postpartum mothers in western countries have shown it to be $10 \%$ to $24 \%$ (11-13). Among the Asian countries, the prevalence was $23 \%$ in India (14) while it was $32.1 \%$ in Sri Lanka (9). The studies carried out to asses PPD of mothers whose neonates were in neonatal intensive care units (NNICU) found out that the rate of PPD increased by $70 \%$ in USA (15), $35.6 \%$ in California (16), $70 \%$ in Ghana (17) and $25 \%$ in Turkey (18).

Mothers' mental health is crucial when their neonates are sick because of the importance of attachment and bonding with the baby; their participation in care; and long-term infant development. The birth of a critically ill infant may cause a crisis for the family. Most of them have some difficulty in adapting to both the hospital environment and especially the NNICU environment. Both these environments are stressful for the parents. Therefore, it is important to find out whether there is an association between neonatal sepsis and PPD of mothers. This would be useful to improve the management of neonatal sepsis and be proactive in identifying women at risk and provide appropriate counselling. The objective of this study was to determine the effect of neonatal sepsis on the PPD of mothers in secondary and tertiary care hospitals in the district of Gampaha.

\section{Methods}

This was a descriptive study conducted in secondary and tertiary care hospitals in Gampaha District in Sri Lanka. The study population for the exposure group was postpartum mothers with neonates who were diagnosed with sepsis. The non-exposure group was postpartum mothers with neonates who were not diagnosed with sepsis and who were born on the same date or within two days of birth of each case. Neonatal sepsis was defined by the presence of one of the clinical signs according to the World Health Organisation Integrated Management of Childhood Illness (IMCI) strategy (19) or culture positive of blood or cerebrospinal fluid. The exclusion criteria were neonates with congenital abnormalities and neonates whose mothers were not residing in Gampaha District. The study setting for the exposure group was four hospitals in this district, namely Colombo North Teaching Hospital, District General Hospital Gampaha, District General Hospital Negombo and Base Hospital Wathupitiwala. The study setting for the controls was from the administrative district of Gampaha.

The sample size was calculated considering an odds ratio (OR) of 1.7 , type I error of 0.05 , power of 0.8 and proportion of PPD among the controls of 0.32 (9). Accordingly, the final sample size was 240 in each exposure and non-exposure groups. The exposure group of mothers was recruited consecutively from the hospitals. The non-exposure group of postpartum mothers was selected from the community in relation to the medical officer of health $(\mathrm{MOH})$ areas where the exposure mothers resided.

The data collection instruments were the Edinburgh postnatal depression scale (EPDS) and an interviewer-administered questionnaire (IAQ). The EPDS has been widely evaluated in many countries and cultures around the world. It has been validated in western countries such as Netherlands (20), USA (21) 
and Sweden (22). It focuses on cognitive and affective symptoms, which avoid the inflation of prevalence confounded by the presence of physical symptoms (such as fatigue and physical discomfort) typical in normal pregnancy and the postpartum period (23). The EPDS consisted of 10 questions. Each question is scored from 0 to 3, making the overall range between 0 and 30. It is a self-administered questionnaire and validated for Sri Lankan context and recommended for use in Sri Lanka. A cut-off score of nine or more detects PPD with $89.9 \%$ sensitivity and $78.9 \%$ specificity $(9,24-25)$. It was concluded that the EPDS scale can be used in the first week of the postpartum period of mothers (26).

The IAQ was designed to assess the associated factors described in other studies $(9,14)$ in relation to PPD. The questionnaire was designed to cover factors of PPD in mothers, such as socio-demographic factors, maternal and neonatal related factors, social support received, major life events experienced and economic hardship. The questions were designed in a simple way and worded in simple language that could be understood easily by the participants. The face and content validity, appropriateness and the relevance in the local context of the IQA were assessed by experts. A pre-test of the IAQ was done to assess the acceptability, comprehension and ease of administration of the questionnaire.

Three pre-intern medical graduates were recruited and trained as research assistants to collect data. It is shown that it would take at least 72 hours to change the psychological status of the mother after sickness of the neonate (26). Owing to this fact, both IAQ and EPDS were administered five days after the diagnosis of neonatal sepsis. For the non-exposure group, it was assessed within two weeks of recruitment.

Support for day-to-day work was assessed by inquiring whether someone was helping in routine activities at home such as cooking, washing, and cleaning. Someone to look after other children was assessed by asking about the people caring for the other kids at home when she was attending on the newborn baby. The experience of abuse meant any kind of abuse including physical, mental, and sexual harassments, and verbal abuses either by the husband or any other family member. The suffering of major illnesses was assessed by asking anyone in the family about facing major accidents, recent stroke, fractured bones, underwent surgery or newly diagnosed cancer or had handicapped children. An unexpected event was defined as death of a close family member. Major economic hardships were assessed based on the loss of occupation of husband or herself, loss of major property, major disasters during the last one year and difficulty in repayment of loans.

\section{Data analysis}

Statistical analysis was conducted employing the software package SPSS (version 16). Bivariate cross tabulations were done to identify the association of PPD with selected socio-demographic factors, maternal factors and neonatal factors. Odds Ratio (OR) and $95 \%$ confidence intervals (CI) were calculated. The factors that showed associations of probability value of $<0.20$ in the bivariate analysis were entered into the multivariate logistic regression model.

\section{Results}

Out of the 240 mothers, four mothers did not respond to the EPDS. Hence, the response rate was $99.2 \%$. The mean age of the study participants was 28 years $(\mathrm{SD}=5$ years). Of the total, $29(6.0 \%)$ mothers were below 20 years and $46(9.6 \%)$ more than 35 years of age. The majority of mothers $(n=446 ; 93.1 \%)$ were Sinhalese and married 473 (98.6\%). The highest proportion $(n=384 ; 80 \%)$ of mothers were not on paid employment.

There were 185 (78.4\%) depressed mothers among the exposure group and $51(21.2 \%)$ among the controls (Figure 1). The association between PPD and neonatal sepsis was statistically significant $(\mathrm{OR}=13.44$; 95\% CI $=8.68,20.83$ ) (Table 1).

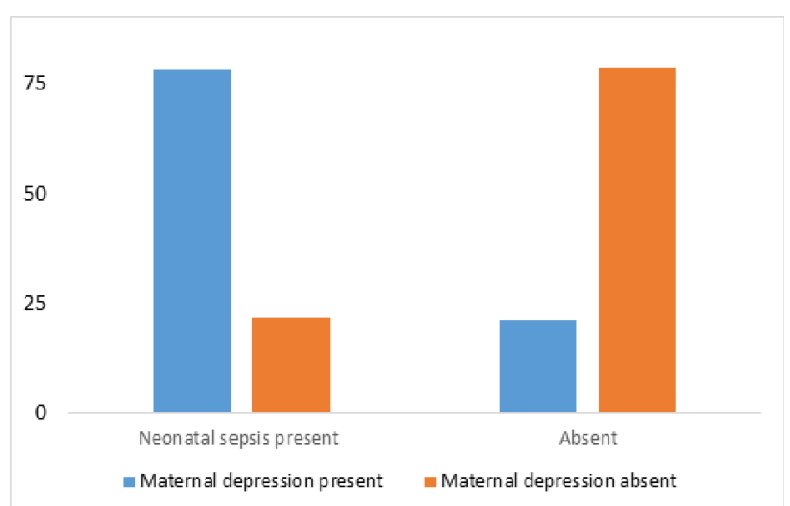

Figure 1. Association of postpartum depression with neonatal sepsis 
All the socio-demographic factors were not significantly associated with the PPD (Table 1). Mothers of babies whose maturity was less than 36 weeks of gestation were significantly more depressed than mothers whose neonates were 36 weeks or more weeks of gestation. Mothers having neonates with a birth weight less than $2500 \mathrm{~g}$ were two times more likely to be depressed than mothers with neonates of a birth weight of more than $2500 \mathrm{~g}$ (Table 2).

There was no significant association of maternal depression and factors such as social support, abuses, major life events, economic hardship and unexpected events in their life (Table 3 ).

Neonatal sepsis was significantly $(\mathrm{OR}=14.0 ; 95 \%$ $\mathrm{CI}=8.6,22.8)$ associated with postpartum depression after controlling the confounding effects (Table 4). Therefore, neonatal sepsis was a major determinant for PPD. Other factors such as the birth weight of the neonate, the maturity of neonates, and bad obstetric history of the mothers were not found significant in multivariate logistic regression analysis.

Table 1. Association of postpartum depression with socio demographic factors of mothers

\begin{tabular}{|c|c|c|c|c|c|c|}
\hline \multirow{3}{*}{ Characteristic } & \multicolumn{4}{|c|}{ Maternal depression } & \multirow{3}{*}{$\begin{array}{c}\text { OR } \\
(95 \% \mathrm{CI})\end{array}$} & \multirow{3}{*}{$p$ value } \\
\hline & \multicolumn{2}{|c|}{ Present $(n=236)$} & \multicolumn{2}{|c|}{ Absent $(n=240)$} & & \\
\hline & No. & $\%$ & No. & $\%$ & & \\
\hline \multicolumn{7}{|l|}{ Age of mothers } \\
\hline$<20$ years & 14 & 48.3 & 15 & 51.7 & 0.95 & 0.88 \\
\hline$\geq 20$ years & 222 & 49.7 & 225 & 50.3 & $(0.45-2.01)$ & \\
\hline \multicolumn{7}{|l|}{ Ethnicity } \\
\hline Non-Sinhalese & 15 & 45.5 & 18 & 54.5 & 0.84 & 0.62 \\
\hline Sinhalese & 221 & 49.9 & 222 & 50.1 & $(0.41-1.70)$ & \\
\hline \multicolumn{7}{|l|}{ Religion } \\
\hline Non-Buddhist & 44 & 42.3 & 60 & 57.7 & 0.69 & 0.09 \\
\hline Buddhist & 192 & 51.6 & 180 & 48.4 & $(0.44-1.07)$ & \\
\hline \multicolumn{7}{|l|}{ Educational level } \\
\hline$<\mathrm{O} / \mathrm{L}$ & 36 & 55.4 & 29 & 44.6 & 1.31 & 0.31 \\
\hline$\geq \mathrm{O} / \mathrm{L}$ & 200 & 48.7 & 211 & 51.3 & $(0.77-0.22)$ & \\
\hline \multicolumn{7}{|c|}{ Mother's occupation } \\
\hline Housewife & 186 & 48.9 & 194 & 51.1 & 0.88 & 0.58 \\
\hline Paid occupation & 50 & 52.1 & 46 & 47.9 & $(0.56-1.38)$ & \\
\hline \multicolumn{7}{|l|}{ Income (Rupees) } \\
\hline$\leq 10000$ & 11 & 35.5 & 20 & 64.5 & 0.54 & 0.10 \\
\hline $10,001-$ & 225 & 50.6 & 200 & 49.4 & $(0.25-1.15)$ & \\
\hline
\end{tabular}


Table 2. Association of postpartum depression and maternal and neonatal factors

\begin{tabular}{|c|c|c|c|c|c|c|}
\hline \multirow{3}{*}{ Characteristic } & \multicolumn{4}{|c|}{ Maternal depression } & \multirow{3}{*}{$\begin{array}{c}\text { OR } \\
(95 \% \mathrm{CI})\end{array}$} & \multirow{3}{*}{ p value ${ }^{1}$} \\
\hline & \multicolumn{2}{|c|}{ Present $(n=236)$} & \multicolumn{2}{|c|}{ Absent $(n=240)$} & & \\
\hline & No. & $\%$ & No. & $\%$ & & \\
\hline \multicolumn{7}{|l|}{ POA } \\
\hline$<36$ weeks & 38 & 84.4 & 7 & 15.6 & 6.27 & 0.001 \\
\hline$\geq 36$ weeks & 199 & 46.4 & 230 & 53.3 & $(2.74-14.36)$ & \\
\hline \multicolumn{7}{|c|}{ Presence of bad obstetric history } \\
\hline Present & 42 & 62.5 & 25 & 37.3 & 1.86 & 0.01 \\
\hline Absent & 194 & 47.4 & 215 & 52.6 & $(1.09-3.17)$ & \\
\hline \multicolumn{7}{|l|}{ Gender of the neonates } \\
\hline Male & 129 & 52.0 & 119 & 48.0 & 1.23 & 0.27 \\
\hline Female & 107 & 46.9 & 121 & 53.1 & $(0.85-1.76)$ & \\
\hline \multicolumn{7}{|c|}{ First born or subsequent born } \\
\hline Being a First born & 128 & 53.3 & 111 & 46.4 & 1.38 & 0.08 \\
\hline Subsequent children & 108 & 45.6 & 129 & 54.4 & $(0.96-1.98)$ & \\
\hline \multicolumn{7}{|l|}{ Birth weight } \\
\hline$<2500$ & 62 & 64.6 & 34 & 35.4 & 2.61 & 0.001 \\
\hline$\geq 2500$ & 174 & 45.8 & 206 & 54.4 & $(1.36-3.44)$ & \\
\hline \multicolumn{7}{|l|}{ Age of onset of sepsis } \\
\hline$\geq 72$ hours & 54 & 72.0 & 21 & 28.0 & 1.69 & 0.1 \\
\hline$<72$ hours & 131 & 81.4 & 30 & 18.6 & $(0.89-3.24)$ & \\
\hline \multicolumn{7}{|l|}{ Type of the hospital } \\
\hline Tertiary care hospital & 136 & 49.3 & 140 & 50.7 & 1.05 & 0.79 \\
\hline Secondary care hospital & 101 & 50.5 & 99 & 49.5 & $(0.73-1.51)$ & \\
\hline
\end{tabular}

${ }^{1}$ Significant at $\mathrm{p}<0.05$ given in bold letters 
Table 3. Association of postpartum depression and maternal and social support, major life events, economic hardship and abuse

\begin{tabular}{|c|c|c|c|c|c|c|}
\hline \multirow{3}{*}{ Characteristic } & \multicolumn{4}{|c|}{ Maternal depression } & \multirow{3}{*}{$\begin{array}{c}\text { OR } \\
(95 \% \mathrm{CI})\end{array}$} & \multirow{3}{*}{ p value ${ }^{1}$} \\
\hline & \multicolumn{2}{|c|}{ Present } & \multicolumn{2}{|c|}{ Absent } & & \\
\hline & No. & $\%$ & No. & $\%$ & & \\
\hline \multicolumn{7}{|l|}{ Social support } \\
\hline Absent & 3 & 60.0 & 2 & 40.0 & 1.51 & 0.65 \\
\hline Present & 234 & 49.8 & 236 & 50.2 & $(0.25-9.14)$ & \\
\hline \multicolumn{7}{|c|}{ Support during hospital stay } \\
\hline Yes & 3 & 60.0 & 2 & 40.0 & 1.51 & 0.65 \\
\hline No & 234 & 49.8 & 236 & 50.2 & $(0.25-9.14)$ & \\
\hline \multicolumn{7}{|l|}{ Abuse of mother } \\
\hline Yes & 3 & 100.0 & 0 & 0.0 & 0.49 & 0.08 \\
\hline No & 234 & 49.5 & 239 & 50.5 & $(0.45-0.54)$ & \\
\hline \multicolumn{7}{|c|}{ Major illness among family members } \\
\hline Yes & 2 & 50.0 & 2 & 50.0 & 1.01 & 0.99 \\
\hline No & 235 & 49.8 & 237 & 50.2 & $(0.14-7.22)$ & \\
\hline \multicolumn{7}{|c|}{ Having economic hardship } \\
\hline Yes & 6 & 100.0 & 0 & 0.0 & 0.49 & 0.01 \\
\hline No & 230 & 49.0 & 239 & 51.0 & $(0.45-0.54)$ & \\
\hline \multicolumn{7}{|c|}{ Experience of unexpected events } \\
\hline Yes & 5 & 71.4 & 2 & 28.6 & 2.57 & 0.25 \\
\hline No & 231 & 49.4 & 237 & 50.6 & $(0.49-13.35)$ & \\
\hline
\end{tabular}

${ }^{1}$ Significant at $\mathrm{p}<0.05$ given in bold letters 
Table 4. Multivariate logistic regression model for maternal depression and factors

\begin{tabular}{|c|c|c|c|c|c|c|c|}
\hline Variables & B & SE & Wald & OR & & CI & $\begin{array}{c}\text { p } \\
\text { value }^{1}\end{array}$ \\
\hline Presence of neonatal sepsis & 2.64 & 0.25 & 113.7 & 14.0 & 8.68 & 20.83 & 0.00 \\
\hline Birth weight $<2500 \mathrm{~g}$ & -0.19 & 0.34 & 0.33 & 0.82 & 0.43 & 1.59 & 0.56 \\
\hline Maturity of neonate $<36$ weeks & 0.75 & 0.51 & 2.16 & 2.12 & 0.78 & 5.70 & 0.14 \\
\hline $\begin{array}{l}\text { Bad obstetric history (history of } \\
\text { abortions, still birth, neonatal death) }\end{array}$ & -0.49 & 0.33 & 2.18 & 0.61 & 0.31 & 1.18 & 0.14 \\
\hline Constant & 1.25 & 0.16 & 61.3 & 0.29 & & & 0.00 \\
\hline
\end{tabular}

B-beta coefficient; SE-standard error; OR- odds ratio; CI-confidence interval

${ }^{1}$ Significant at $\mathrm{p}<0.05$ given in bold letters

\section{Discussion}

In the present study, it was revealed that the presence of PPD among the mothers of neonatal sepsis neonates was $78.4 \%$, whereas PPD among the control group was $21.6 \%$. It was statistically significant. Another Sri Lankan study also reported that illness of the neonate was significantly associated with PPD (9).

One study carried out in Turkey revealed using the EPDS that $29.5 \%$ of NNICU group mothers were depressed whereas in the control group it was $13.6 \%$ $(p<0.005)(27)$. But in this study, the cutoff level for EPDS score was 13. The difference of PPD among the mothers observed could be due to different cutoff values used in EPDS in different studies $(6,28)$. Another study carried out at a California tertiary care NNICU found that $77.8 \%$ of mothers were positive for symptoms of depression and $35.6 \%$ of mothers were PPD (16). Using the Patient Health Questionnaire for assessing depression among the mothers of hospitalized infants in NNICU, it was found that $70 \%$ of mothers had PPD (17). Another study used the Beck Depression Inventory for assessing postpartum depression among mothers who delivered premature neonates in Nigeria found that $15.1 \%$ of mothers were depressed with preterm neonates in NNICU than the term neonates (3.7\%) (29). The actual proportions of depressed mothers were difficult to compare in different studies due to the use of different scales and tools $(17,29)$.
According to the findings of the current study, there was no association between any of the sociodemographic factors and PPD. Similar findings were revealed by others $(27-28,30)$ that there was no significant association between socio-demographic factors namely maternal age, education level of mothers, and working status of mothers and PPD of mothers of neonates admitted to NNICU. However, one study reported that unemployment was associated with PPD (31).

According to the bivariate analysis, low birth weight and preterm birth were associated with PPD. Similar to our findings, another study reported that low birth weight was not independently associated with PPD (27) In contrast, two studies reported that low weight or preterm birth was independently associated with postpartum depression (32). We could not find any association between the experience of unexpected life events and PPD. In contrast to our findings, three studies reported that life events increased the risk of PPD $(9,31,33)$. According to our study, there was no association between abuse of mother and PPD. In contrast, two studies reported that poorer relationships with husbands or parents (34), and unstable marital condition (35) were associated with postpartum depression.

The difference in findings may be due to the study settings in which they were carried out, the former 
study was carried out among postpartum mothers in the community, whereas the present study was carried out in the hospitals and from controls in the community. Other main differences between the two studies were the time of administration of the EPDS questionnaire. The EPDS questionnaire was administered after six weeks of the postnatal period in the former study whereas current study EPDS administered within two weeks of delivery to postpartum mothers.

There are few limitations inherent to our study design. The sensitivity and specificity of the EPDS are not optimal (24). Therefore, the detection of postpartum depression was subjected to some misclassification. Further, diagnosis of neonatal sepsis was based on IMCI criteria which has a sensitivity of $85 \%$ and a specificity of $75 \%$ (19), in addition to considering the culture positive status. Still, there was a risk of false negative diagnosis which leads to misclassification bias. Third, the selection of the study population based on classification on the neonatal sepsis which leads to overestimating the association with PPD. To prevent this selection bias, we recommend consecutive sampling of postpartum mothers from hospitals and following them up for a reasonable period of time.

\section{Conclusions \& Recommendations}

PPD among mothers with neonatal sepsis babies was $78 \%$ compared to $28 \%$ among mothers of nonsepsis babies in the district of Gampaha. It is timely to introduce a screening programme to detect postpartum depression among mothers whose neonates are at neonatal units. Thereafter, curative, supportive and counselling services for them can be arranged. Routine psychiatric support services should be improved for these mothers. Institutional staff in postnatal wards and NNICU need to be trained on managing PPD.

\section{Public Health Implications}

Neonatal sepsis is strongly associated with postpartum depression. Screening and management of postpartum depression is essential in clinical practice.

\section{Author Declarations}

Competing interests: The authors declare that they have no conflicts of interests' in this study.

Ethics approval and consent to participate: The ethics clearance was obtained from the Ethics Review Committee of the Faculty of Medicine, University of Kelaniya. Informed written consent was obtained from all the participants.

\section{Funding: Self-funded}

Acknowledgements: We are grateful to the Postgraduate Institute of Medicine, University of Colombo, the data collectors and the participants for the study.

Author contributions: CJ participated in the design of the study, coordinated data collection, performed the statistical analysis and drafted the first version of the manuscript. CA participated in the design of the study, performed the statistical analysis, interpreted the data and helped to draft the manuscript. Both authors read and approved the final manuscript.

\section{References}

1. Miles MS, Funk SG, Carlson J. Parental Stressor Scale: neonatal intensive care unit. Nursing Response 1993; 42(3): 148-152.

2. Shields PD \& Pinelli J. Variables associated with parental stress in neonatal intensive care units. Neonatal Network 1997; 16: 29-37.

3. Chourasia N, Surianarayan P, Adhisivam B, Bhat V. NICU admission and maternal stress levels. Indian Journal of Paediatrics 2013; 80(5): 380-384.

4. Szczapa T, Gadzinowska-Szczucinska J, Jopec A. Evaluation of stress responses in parents of newborns hospitalized in the intensive care unit. Ginekologia Polska 2009; 80(5): 367-373.

5. Tamaki R, Murata M, Okano T. Risk factors for postpartum depression in Japan. Psychiatry Clinical Neuroscience 2008; 51(3): 93-98.

6. Adewuya AO, Fatoye FO, Ola BA, Ijaodola OR., Ibigbami SM. Socio demographic and obstetric risk factors for postpartum depressive symptoms in Nigerian women. Journal of Psychiatric Practice 2005; 11(5): 353-358.

7. Carl C Bell. Diagnostic and Statistical Manual of Mental Disorders (DSM-IV). Washington DC: American Psychiatric Association, 1994. 
8. Beck CT. Predictors of postpartum depression: an update. Nursing Research 2001; 50(5): 275-285.

9. Rowel DDS. Prevalence, incidence and correlates of postpartum depression in the Puttalam district. MD Thesis (Community Medicine). Colombo: Postgraduate Institute of Medicine, 2004.

10. Robertson E, Grace S, Wallington T, Stewart DE. Antenatal risk factors for postpartum depression: a synthesis of recent literature. General Hospital Psychiatry 2004; 26(4): 289-295.

11. Pitt B. Atypical depression following childbirth. British Journal of Psychiatry 1968; 114(516): 1325-1335.

12. Benvenutti P, Ferrara M, Niccolai C, Valoriani V, Cox J. The Edinburgh Postnatal Depression Scale: validation on an Italian sample. Journal of Affective Disorders 1999; 53(2): 137-141.

13. Areias MBG, Kumar R, Barro HS, Figueiedo E. Correlate of postnatal depression in mothers and fathers. British Journal of Psychiatry 1996; 169(1): 36-41.

14. Patel V, De Souza N, Rodrigues M. Postnatal depression and infant growth and development in low income countries: a cohort study from Goa, India. Archives of Disease in Childhood 2003; 88(1): 34-37.

15. Mount KO. Screening for maternal depression in the neonatal ICU. Clinical Perinatology 2009; 36(1): 137-152.

16. Shaw RJ, Lilo EA, Storfer-Isser A, Ball MB, Proud MS, Vierhaus NS, et al. Screening for symptoms of postpartum traumatic stress in a sample of mothers with preterm infants. Issues Mental Health Nursing 2014; 35(3): 198-207.

17. Gold JK, Spangenberg K, Wobil P, Schwenk Tl. Depression and risk factors for depression among mothers of sick infants in Kumasi, Ghana. International Journal of Gynaecology and Obstetrics 2013; 120(3): 228-231.

18. Akman K, Kus CN, Zdemir O. Mothers' postpartum psychological adjustment and infantile colic. Archives of Disease in Childhood 2006; 91(5): 417-419.

19. WHO. Handbook: Integrated Management of Childhood Illness. Geneva: World Health Organization, 2005.

20. Pop VJ, Komproe IH, Vanson MK. Characteristics of Edinburg Postnatal Depression Scale in Netherlands, Journal of Affective Disorders 1992; 26(2): 105-110.

21. Schaper AM, Rooney BL, Kay NR, Silva PD. Use of the Edinburgh Postnatal Depression Scale to identify postpartum depression in a clinical setting. Journal of Reproductive Medicine 1994; 39(8): 620-624.
22. Alvarado-Esquivel C, Sifuentes-Alvarez A, SalasMartinez C. The use of the Edinburgh Postpartum Depression Scale in a population of teenager pregnant women in Mexico: a validation study. Clinical Practice \& Epidemiology in Mental Health 2014; 10 : 129-132.

23. Regmi S, Sligli W, Carter D, Grut W, Seear M. A controlled study of postpartum depression among Nepalese women: validation of the Edinburg Postpartum Depression Scale in Kathmandu. Tropical Medicine \& amp; International Health 2002; 7(4): 378-382.

24. Rowel D, Jayawardena P, Fernando N. Validation of the Sinhala Translation of Edinburgh Postnatal Depression Scale. Ceylon Medical Journal 2007; 53(1): $10-15$.

25. Chandran M, Tharyan P, Mulliyal J, Abraham S. Postpartum depression in a cohort of women from a rural area of Tamil Nadu, India. Incidence and risk factors. British Journal of Psychiatry 2002; 181 : 499-504.

26. Teissedre F \& Chabrol H. A study of Edinburgh Postnatal Depression Scale (EPDS) on 859 mothers: detection of mothers at risk for postpartum depression. L'Encephale 2004; 30(4): 316-381.

27. Yurdakul Z, Akman I, Kuskun KM, Karabekiroglu A, Yaylali G, Demir F, Ozek E. Maternal psycological problems associated with national intensive care admission. International Journal of Paediatrics 2009; 7: $18-25$.

28. Charter JD, Mulder RT, Bartram AF, Darlow BA. Infants in a neonatal intensive care unit: parental response. Archives of Disease in Childhood. Fetal \& Neonatal Edition 2005; 90(2): F109-F113.

29. Ukpong DI, Fatoye FO, Oseni SB, Adewuya AO. Postpartum emotional distress in mothers of preterm infants: a controlled study. East African Medical Journal 2003; 80(6): 289-292.

30. Ayoub K, Shaheen A, Hajat S. Prevalence and associated factors of post-partum depression in Palestinian mothers: a cross-sectional study. The Lancet 2017; 390: S31.

31. Sulyman D, Ayanda KA, Dattijo LM, Aminu BM. Postnatal depression and its associated factors among Northeastern Nigerian women. Annals of Tropical Medicine \& Public Health 2016; 9(3): 184190.

32. Silverman ME, Reichenberg A, Savitz DA, Cnattingius S, Lichtenstein P, Hultman CM, Larsson H, Sandin S. The risk factors for postpartum depression: a 
population-based study. Depression \& Anxiety 2017; 34(2): 178-187.

33. Rathnayake LVR. Maternal depression during postpartum period in the district of Gampaha: prevalence, risk factors and effect on the growth and development of the infants. MD thesis (Community Medicine). Colombo: Postgraduate Institute of Medicine, 2011.
34. Chi X, Zhang P, Wu H, Wang J. Screening for postpartum depression and associated factors among women in China: a cross-sectional study. Frontiers in Psychology 2016; 7: 1668.

35. Kerie S, Menberu M, Niguse W. Prevalence and associated factors of postpartum depression in Southwest, Ethiopia 2017: a cross sectional study. BMC Research Notes 2018; 11: 623. 